\title{
Effects of Training on a Spatial Memory Task on High Affinity Choline Uptake in Hippocampus and Cortex in Young Adult and Aged Rats
}

\author{
Michael W. Decker, ${ }^{1}$ Mary Ann Pelleymounter, ${ }^{1}$ and Michela Gallagher ${ }^{1,2}$ \\ 'Curriculum in Neurobiology and 'Department of Psychology, University of North Carolina at Chapel Hill, Chapel Hill, North \\ Carolina 27514
}

\begin{abstract}
The relation of forebrain cholinergic function to learning and memory was explored by identification and characterization of a training-induced change in high-affinity choline uptake (HACU), an index of cholinergic actlvity. Young adult rats were trained to find an invisible escape platform in a water tank using environmental cues. After $4 \mathrm{~d}$ of this place-training ( 16 trials), hippocampal HACU was significantly reduced relative to that observed in rats trained to find a visible platform (cue-training), even when cue- and place-trained rats were yoked for swim time. These place- but not cue-trained rats showed significantly lower hippocampal HACU than did naive rats, and no effect of training was noted after only 1 d of training. Similar results were obtained in parietal cortex. These differential training effects on HACU correspond to previous reports that muscarinic blockade impairs place, but not cue, learning. $A$ further experiment revealed that the decrease in HACU in hippocampus, but not in parietal cortex, occurred only during the acquisition phase of learning and was related to the rate of acquisition for individual animals. Hippocampal HACU in naive young and aged (24-27 months) rats did not differ, but the response of the septohippocampal cholinergic system to training was diminished in the aged rats. Old rats displayed impaired place learning and a corresponding dampening of the training-induced change in HACU. These results suggest that there is a task-specific engagement of cholinergic function in young animals that does not occur in behaviorally impaired aged animals, a finding that is consistent with a role for cholinergic dysfunction in memory impairments associated with aging.
\end{abstract}

A major focus in the study of aging in recent years has been the proposition that cholinergic dysfunction underlies many of the learning and memory deficits associated with aging and with Alzheimer's disease (AD). This cholinergic hypothesis of geriatric memory dysfunction (Bartus et al., 1982) is supported by studies demonstrating impairments in the cholinergic system both in $\mathrm{AD}$ and during normal aging, although the evidence for cholinergic dysfunction is clearly stronger for $\mathrm{AD}$ than for nor-

\footnotetext{
Received Jan. 9, 1987; revised Apr. 27, 1987; accepted July 8, 1987.

This work was supported by NIMH Grants MH39180 and K02 MH00406 to M.G. and by an NSF predoctoral fellowship to M.W.D. The authors wish to thank R. Rosenberg and R. Cody for technical assistance and Dr. R. Mailman for use of laboratory facilities for some of the biochemistry.

Correspondence should be addressed to Michela Gallagher, Ph.D., Dept. of Psychology, Davie Hall 013A, University of North Carolina, Chapel Hill, NC 27514.

Copyright (C) 1988 Society for Neuroscience $0270-6474 / 88 / 010090-10 \$ 02.00 / 0$
}

mal aging (Perry, 1980). A second line of evidence in support of this geriatric cholinergic hypothesis is provided by a growing number of studies demonstrating that experimental disruption of cholinergic function produces impaired performance on a number of learning and memory tasks (for discussion, see Squire and Davis, 1981, and Gold and Zornetzer, 1983). Further, the disturbed performance associated with these cholinergic manipulations is often described as resembling that occurring in older animals (Bartus et al., 1982, 1985). These 2 lines of evidence are consistent with the cholinergic hypothesis of geriatric memory dysfunction, but they do not provide evidence of a direct link between age-related cholinergic impairments and agerelated learning and memory deficits.

A more direct relationship between cholinergic dysfunction and learning and memory impairments associated with aging is difficult to establish. Another approach to this problem has been the attempt to improve the performance of aged animals using pharmacological manipulations designed to enhance cholinergic function. Bartus et al. $(1982,1985)$ note that such treatments have occasionally been successful in diminishing age-related behavioral deficits in experimental animals. This observation, however, does not necessarily imply that the reason for the original learning and memory deficit was impaired cholinergic function, as Bartus et al. $(1982,1985)$ point out that behavioral improvements are sometimes also obtained by enhancing cholinergic neurotransmission in young animals. Behavioral deficits in old animals can also be enhanced by the transplantation of fetal brain tissue enriched in cholinergic neurons (Gage et al., 1984a; Gage and Björklund, 1986). However, the transplanted tissue also contains neurons that use other transmitters and may also contain growth factors and other, unknown agents that might enhance performance. Thus, this phenomenon of improved performance in old animals following cholinergic manipulations provides some support for the cholinergic hypothesis, but falls short of establishing a direct link between the age-related deficits in cholinergic function and impaired learning and memory.

If cholinergic activity is important for normal functioning on a particular learning and memory task, it is reasonable to expect that behavioral training on such a task might be accompanied by some relevant change in cholinergic activity. If such a link can be established between behavioral training and altered cholinergic activity in young animals, a more direct test of the cholinergic hypothesis would be to examine this same relationship in behaviorally impaired old animals.

Recently, Wenk et al. (1984) provided evidence of a training- 
induced modification of cholinergic activity in young animals. They found that, following training on several tasks, rats displayed increased in vitro high-affinity choline uptake (HACU) in hippocampus but not in frontal cortex. Since HACU in cortex and hippocampus is an index of cholinergic activity in these regions (Atweh et al., 1975; Simon and Kuhar, 1975; Simon et al., 1976), Wenk ct al. (1984) interpreted their findings as demonstrating a training-induced change in the activity of the septohippocampal cholinergic system in young animals. In this paper we have taken a similar approach both to further characterize the effect of behavioral training on HACU in young animals and to explore the relationship between behavioral training and $\mathrm{HACU}$ in aged animals.

To study behaviorally related changes in HACU, we measured HACU in hippocampus and in cortex following training in the Morris water maze (Morris, 1981). We selected this task rather than those used by Wenk et al. (1984) because there are 2 different versions of the water maze task, which differ in their sensitivity to disruption by both cholinergic blockade and aging. In this task, rats are placed in a large pool of water in which a single escape platform is located. In the place version of the task, rats learn to navigate to a hidden platform that is consistently placed in the same spatial location from trial to trial. In the cue version, the platform itself is visible to the rat. Thus, in the cue version, the rat can locate the platform merely by approaching a visible cue, but in the place version, rats must locate the platform by learning its position in relation to the configuration of extramaze cues. Training on the place version of this task is disrupted by hippocampal damage (Morris et al., 1982; Sutherland et al., 1983), muscarinic blockade (Sutherland et al., 1982; Whishaw, 1985; Buresova et al., 1986), and by lesions of the nucleus basalis magnocellularis (nBM), the major source of extrinsic cholinergic input to cortex (Dokla et al., 1985; Whishaw et al., 1985). Also, place-training is impaired in senescent rats (Gage et al., 1984b; Rapp et al., 1987). In contrast, training on the cue version of the task is not significantly disrupted by nBM lesions (Dokla et al., 1985), hippocampal lesions (Morris et al., 1982), or muscarinic blockade (Whishaw, 1985). Further, the effects of aging on acquisition of the cue version of the task appear to be less than those in the place version (Rapp et al., 1987).

In that the cue and place versions of the water maze task are differentially sensitive to disruption by cholinergic blockade, we reasoned that the cholinergic system might be differentially affected by training on these tasks. Thus, we predicted that placetraining might produce changes in hippocampal HACU in young rats that would not be seen after cue-training. One set of experiments reported here explores the relation between training on these tasks and HACU in young rats. We then provide evidence that this relationship is altered in aged rats.

An abstract of some of this work has appeared previously (Decker et al., 1986).

\section{Materials and Methods}

Subjects. Male Long-Evans hooded rats, 3-5 or 24-27 months old at the time of initial behavioral testing, were obtained from Charles River Laboratories. Aged rats were retired breeders obtained at approximately 9 months of age and housed under pathogen-free conditions at the University of North Carolina animal care facilities until use. All animals were singly housed in opaque $45 \times 24 \times 20 \mathrm{~cm}$ plastic breeding cages for 1 week prior to and during the entire course of testing. The animal colony was maintained at $25^{\circ} \mathrm{C}$ on a $12 \mathrm{hr}$ light: dark cycle (lights on at 0700 hours). Water and standard rat chow were freely available throughout the experiment.

Apparatus. The water maze has been described previously (Rapp et al., 1987). Briefly, it is a circular, galvanized-steel watering tank measuring $1.83 \mathrm{~m}$ in diameter and $0.58 \mathrm{~m}$ in height, with its interior surface painted white. During testing, the maze was filled to a depth of 35.5 $\mathrm{cm}$ with water clouded by the addition of $0.9 \mathrm{~kg}$ dry milk and kept at a temperature of $26 \pm 1^{\circ} \mathrm{C}$. A cylindrical escape platform measuring $10.2 \mathrm{~cm}$ in diameter was located in 1 of 4 possible positions in the water. Each of these positions was centered in 1 of the 4 quadrants of the pool. For some animals, a white platform (the place-training platform) was positioned such that its top surface remained $1.5 \mathrm{~cm}$ below the surface of the water; for the other animals, a black platform (the cue-training platform) protruded about $1.5 \mathrm{~cm}$ above the water's surfacc.

Behavioral procedures. For 2 weeks prior to training, animals were handled daily. On the day before training, animals were placed in the pool with no escape platform present and allowed to swim for $90 \mathrm{sec}$ to habituate them to the apparatus. Beginning the following day, each animal was placed in the water at 1 of 4 designated entry locations, varied from trial to trial in a quasirandom order, and allowed to swim until the escape platform was found or $120 \mathrm{sec}$ elapsed. On any trial during which the animal failed to find the platform within $120 \mathrm{sec}$, it was placed on the platform by the experimenter. Each animal then spent $60 \mathrm{sec}$ on the platform before being removed to a holding cage for a 60 sec intertrial interval.

Three training conditions were used: cue, place, and yoked control. Cue-trained animals were trained to find the visible black platform that protruded from the water surface. The location of this platform was varied in a quasirandom order such that each of the 4 possible locations was used at least once during a training day. Place-trained animals were required to find the white submerged platform. This platform, while not visible to the animals in the pool, was reliably located in the same position with respect to the external spatial environment on every trial, thereby allowing the animal to determine the platform location by using extramaze cues. Yoked controls were trained exactly as were cue-trained animals, except that the escape platform was not immediately available, but was placed in the tank after a designated period of time corresponding to the escape latencies of place-trained animals. In this way, the amount of time that the yoked controls spent swimming over the course of training was matched to that of place-trained animals.

Three different training schedules were used. Animals received either 6 trials on a single day of training, 4 trials/d for 4 consecutive days, or 4 trials/d for 8 consecutive days. Animals receiving either 4 or $8 \mathrm{~d}$ of training all received a probe trial as the fourth trial on the third training day. The probe trial consisted of placing the animal in the tank with no platform present and allowing the animal to swim freely for $90 \mathrm{sec}$. This trial was videotaped and later analyzed to determine each animal's search pattern. This was accomplished by dividing the tank into 4 quadrants, each containing a potential escape platform position at its center. Using a template so marked and affixed to the video screen, the amount of time each animal spent in each of the 4 quadrants was recorded. In addition, each time an animal crossed through a possible escape platform position, an annulus crossing was recorded for that quadrant of the pool.

Biochemical procedures. HACU was measured using a modification of the proccdurcs of Sherman et al. (1978) and Simon et al. (1976). Animals were decapitated 15 min following the completion of their last training trial and their brains rapidly removed and dissected over ice. The hippocampus and portions of parietal, and in some cases prefrontal, cortex were dissected out. Tissues were weighed and homogenized in 20 volumes of ice-cold $0.32 \mathrm{~m}$ sucrose, using a hand-held Teflon-onglass homogenizer (10-12 strokes). After centrifugation of the homogenate at $1000 \times g$ for $10 \mathrm{~min}$ at $2^{\circ} \mathrm{C}$, the supernatant was spun at $18,000 \times g$ for $20 \mathrm{~min}$ to yield a crude synaptosomal $/ \mathrm{mitochondrial}$ pellet. The pellet was resuspended in 24 volumes of cold $0.32 \mathrm{M}$ sucrose, and $50 \mu \mathrm{l}$ of this suspension (approximately $75 \mu \mathrm{g}$ protein) was added to tubes containing $450 \mu \mathrm{l}$ of ice-cold incubation buffer (final dilution: $126 \mathrm{~mm} \mathrm{NaCl}, 4.75 \mathrm{~mm} \mathrm{KCl}, 1.27 \mathrm{~mm} \mathrm{CaCl}_{2}, 15.8 \mathrm{~mm} \mathrm{Na}_{2} \mathrm{HPO}_{4}, 1.42$ $\mathrm{mM} \mathrm{MgCl}_{2}$, and $2 \mathrm{mg} / \mathrm{ml}$ dextrose; $\mathrm{pH} 7.4$ ), to which ${ }^{3} \mathrm{H}$-choline (final sp act, $4.2 \mathrm{Ci} / \mathrm{mm}$; Ncw England Nuclear) had been added (final dilutions, $0.05-1.6 \mu \mathrm{M})$. Sodium-independent uptake was measured in parallel using incubation buffer in which $\mathrm{NaCl}$ had been replaced with $\mathrm{LiCl}$ and $\mathrm{Na}_{2} \mathrm{HPO}_{4}$ had been replaced with Tris phosphate. Tubes were then placed in a shaking water bath for $2.5 \mathrm{~min}$ at $37^{\circ} \mathrm{C}$, followed by cooling in an ice-water bath. After the addition of $2 \mathrm{ml}$ of ice-cold sodium-free 


\section{UPTAKE AT 0.4UM CHOLINE-HIPPOCAMPUS ONE DAY OF TRAINING}

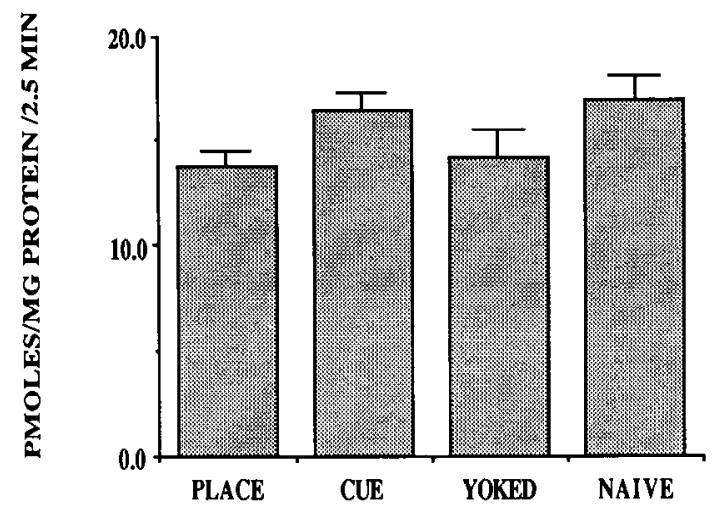

\section{UPTAKE AT 0.4 UM CHOLINE-HIPPOCAMPUS FOUR DAYS OF TRAINING}

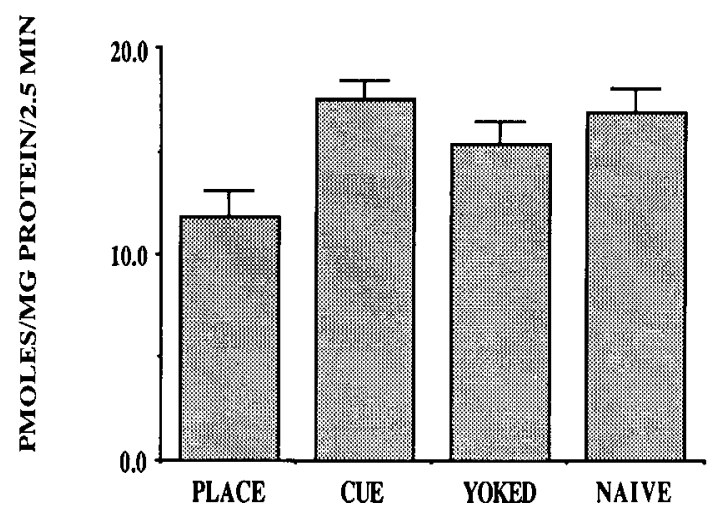

Figure 1. High-affinity choline uptake at $0.4 \mu \mathrm{M}$ choline in hippocampus of behaviorally naive rats $(n=7)$ compared to that in rats trained for $1 \mathrm{~d}$ (above) or for $4 \mathrm{~d}$ (below). Uptake in rats place-trained for $4 \mathrm{~d}$ is significantly lower than that in naive rats $(p<0.01)$, rats cue-trained for $4 \mathrm{~d}$, or yoke-trained rats at $4 \mathrm{~d}(p<0.05)$. One day of training has no significant effect.

buffer, samples were filtered over glass fiber filters (A/E; Gelman). The filters were then rinsed twice with $2 \mathrm{ml}$ of ice-cold buffer and counted in $10 \mathrm{ml}$ of Scintiverse E (Fisher). HACU in all samples was measured in triplicate and determined by subtracting sodium-independent uptake from total uptake. Protein was determined by the method of Lowry et al. (1951). Characterization of HACU using these procedures reveals a $K_{\mathrm{m}}$ of about $0.4 \mu \mathrm{M}$ using Eadie-Hofstee analysis. Uptake is linear with tissue concentration up to at least $140 \mu \mathrm{g}$ protein/tube, and linear with incubation time up to 3 min.

Statistical analysis. Statistical significance was determined using analysis of variance (ANOVA), with evaluation of post hoc pairwise comparisons using Duncan's multiple-range method. In evaluating all comparisons, we used 2-tailed tests of significance.

\section{Results}

\section{Experiment 1}

An initial experiment was conducted to measure baseline levels of HACU in experimentally naive, aged Long-Evans rats, as few studies have sought to measure this variable as a function of age, and no data are available on hippocampal HACU changes with age in this particular strain of rat. Uptake was assessed at 5 concentrations of choline for each of 7 old and 6 young animals to derive estimates of the kinetic parameters $K_{\mathrm{m}}$ and $V_{\max }$. Using
Eadie-Hofstee analysis, we found $K_{\mathrm{m}} \mathrm{s}$ of $0.47 \pm 0.08$ and $0.36 \pm$ $0.04 \mu \mathrm{M}$ and $V_{\max }$ s of $43.83 \pm 6.54$ and $40.79 \pm 3.67 \mathrm{pmol} / \mathrm{mg}$ protein $/ 2.5 \mathrm{~min}$ for old and young rats, respectively. Thus, in contrast to Sherman et al. (1981), who used Fischer 344 rats, we did not observe a significant effect of age on baseline levels of hippocampal HACU in the Long-Evans strain.

As the failure to find an age-related change in baseline levels of HACU does not preclude the possibility that the hippocampal system might be differentially affected by behavioral manipulations in old and young animals, we next sought to characterize the effects of behavioral training on $\mathrm{HACU}$ in young animals so that we might later compare these with the effects obtained in aged rats.

\section{Experiment 2}

Six different groups of trained animals were used, and the HACU measured at a concentration of $0.4 \mu \mathrm{M}$ choline in each treatment condition was compared to that in naive animals for both hippocampus and parietal cortex. These animals received either 1 $\mathrm{d}$ of training (6 trials) or $4 \mathrm{~d}$ of training (16 trials) on 1 of the 3 training procedures - cue, place, or yoked. The effects of training on HACU in the hippocampus are displayed in Figure 1. Comparison of animals in these training groups with behaviorally naive animals was by 1 -way ANOVA. This analysis reveals a significant effect of training on hippocampal $\operatorname{HACU}(F(6,44)=$ $3.55, p<0.01$ ), and pairwise comparisons of each training group to naive animals shows that animals trained for $4 \mathrm{~d}$ on the place version of the Morris water maze displayed significantly lower hippocampal HACU than that of naive animals $(p<0.01)$. At this point in training ( 16 trials), neither the cue-trained nor the yoked control animals differed significantly from naive animals; after $1 \mathrm{~d}$ of training, none of the groups of trained animals differed significantly from naive animals. In addition, after $4 \mathrm{~d}$ of training, place-trained rats displayed significantly lower hippocampal HACU than that of yoked control animals trained for $4 \mathrm{~d}(p<0.05)$, and $4 \mathrm{~d}$ of place-training resulted in significantly lower hippocampal HACU than did $4 \mathrm{~d}$ of cue-training $(p<0.01)$.

These data indicate that training in the Morris water maze affects hippocampal HACU, but, more important, that the type of training determines whether or not there will be an effect. Wenk et al. (1984) found that every training procedure they used altered hippocampal HACU. Our findings, in contrast, reveal a correspondence between the established sensitivity of place-training to cholinergic blockade and to hippocampal damage and its effect on HACU. Conversely, the performance of the cue version of the task, which has been shown to be relatively insensitive to hippocampal damage or cholinergic blockade, does not affect hippocampal HACU. Kinetic analysis of this differential effect of cue- and place-training on HACU, using EadieHofstee analysis of $\mathrm{HACU}$ at 5 concentrations of choline $(0.05$, $0.10,0.20,0.40$, and $0.80 \mu \mathrm{M})$, reveals a significant difference in $V_{\max }(39.66 \pm 3.97$ and $26.17 \pm 4.29 \mathrm{pmol} / \mathrm{mg}$ protein $/ 2.5$ min for cue- and place-trained animals, respectively; $t(16)=$ $2.31, p<0.05$ ) and a suggestive, but not statistically significant, difference in $K_{\mathrm{m}}(0.54 \pm 0.07 \mu \mathrm{M}$ for cue and $0.36 \pm 0.06 \mu \mathrm{M}$ for place, $p>0.05$ ).

Data collected in parallel for parietal cortex (see Fig. 2) reveal a similar pattern. ANOVA reveals a significant $F$ value $(F(6,44)=$ $2.45, p<0.05$ ), and cortical HACU in the training group receiving $4 \mathrm{~d}$ of place-training differs significantly from that measured in naive rats or yoked controls trained for $4 \mathrm{~d}$ controls $(p$ 


\section{UPTAKE AT 0.4UM CHOLINE-CORTEX ONE DAY OF TRAINING}
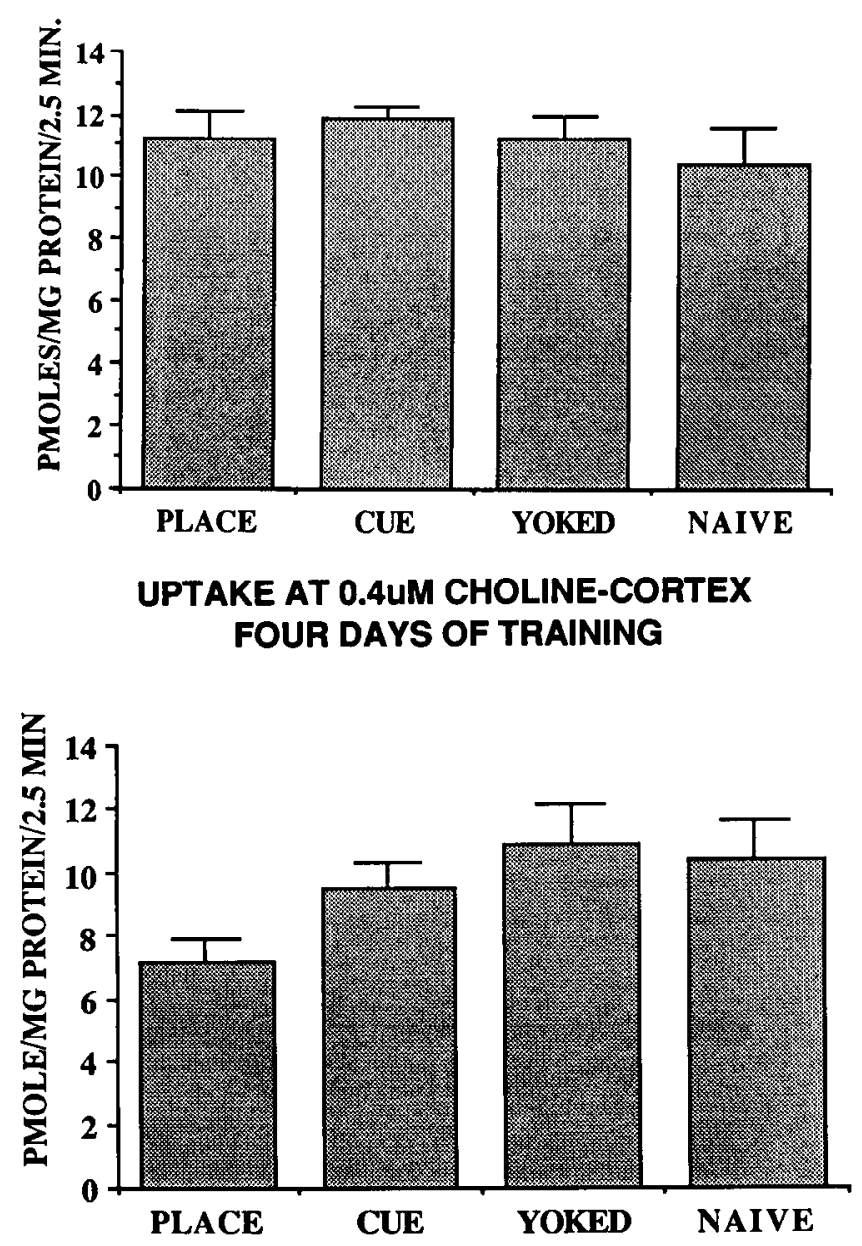

Figure 2. High-affinity choline uptake at $0.4 \mu \mathrm{M}$ choline in parietal cortex of behaviorally naive rats $(n=7)$ compared to that in rats trained for $1 \mathrm{~d}$ (above) or for $4 \mathrm{~d}$ (below). Uptake in rats place-trained for $4 \mathrm{~d}$ is significantly lower than that in naive rats $(p<0.05)$ or yoke-trained rats at $4 \mathrm{~d}(p<0.05)$. One day of training has no significant effect.

$<0.05)$. Unlike in hippocampus, however, the difference in cortical HACU between cue- and place-trained animals was not statistically significant after $4 \mathrm{~d}$ of training. As with hippocampal HACU, cortical HACU was not altered by only $1 \mathrm{~d}$ of training. This similar pattern of results in parietal cortex and hippocampus suggests that training-induced changes in cortical HACU might parallel those observed in hippocampus. This may be due to some covariation in HACU in hippocampus and parietal cortex in individual animals. For example, a relationship between HACU in hippocampus and parietal cortex is present among animals place-trained for $4 \mathrm{~d}(r=0.74, p<0.02)$, and a similar relationship exists between hippocampal and cortical HACU in animals cue-trained for $4 \mathrm{~d}(r=0.69, p<0.05)$.

\section{Experiment 3}

Having established that $4 \mathrm{~d}$ of place-training reduces subsequently measured in vitro HACU in hippocampus in young animals, and further that cue-training does not produce this effect, we now consider the effects of place- and cue-training on HACU in old animals. In this experiment, 9 old rats were trained

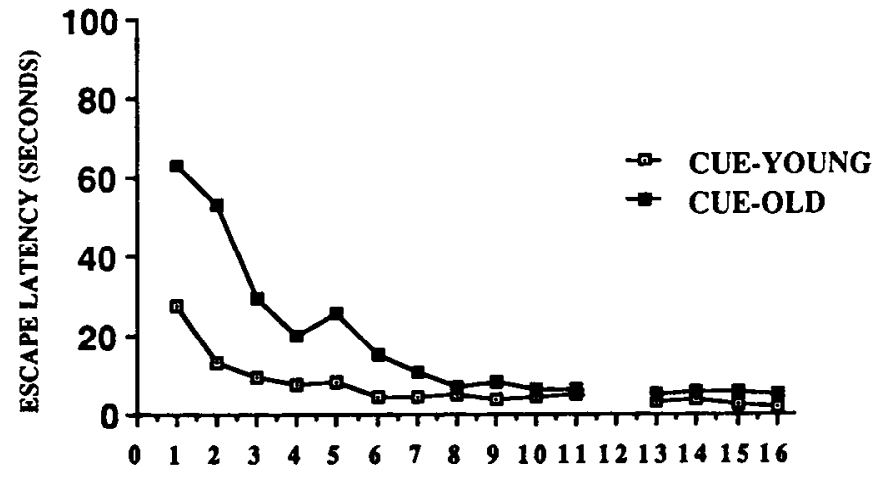

Figure 3. Escape latencies of old and young rats cue-trained for $4 \mathrm{~d}$.

for $4 \mathrm{~d}$ on the place version of the water maze and 8 were cuetrained for $4 \mathrm{~d}$. Training and assays for these old animals were conducted in parallel with those reported for Experiment 2, so the results of Experiments 2 and 3 can be directly compared. One of the 8 cue-trained old animals was excluded from further analysis because necropsy revealed a large pituitary tumor. Comparisons of the escape latencies of these aged animals with those of the cue- and place-trained young animals discussed previously are found in Figures 3 and 4. A 3-way ANOVA (age $\times$ training condition $\times$ trials) with repeated measures for the trials preceding the free swim probe (on trial 12 ) reveals a significant trials effect $(F(10,300)=12.10, p<0.001)$, indicating that animals did learn to escape more quickly with repeated experience, as well as a significant effect of training condition $(F(1,30)=32.67, p<0.001)$, which indicates that animals locate the visible platform more quickly than the invisible platform. The tendency for aged animals to take longer to locate the escape platform does not quite reach statistical significance $(F(1,30)=$ $3.31, p<0.10$ ), and no statistically significant interactions were found. For the trials conducted on the day the animals were killed (trials 13-16), only the main effect of training condition was statistically significant $(F(1,30)=16.96, p<0.001)$, with cue-trained animals again escaping more quickly than placetrained animals.

Analysis of the probe trial free swim data reveals an agerelated behavioral deficit that is not apparent using the escape latency measure. The amount of time spent in each of the 4 quadrants during the $90 \mathrm{sec}$ free swim (no platform available) is depicted for cue- and place-trained young animals in the top portion of Figure 5, and for old animals in the bottom portion. In each case a quadrant has been designated as the "target"

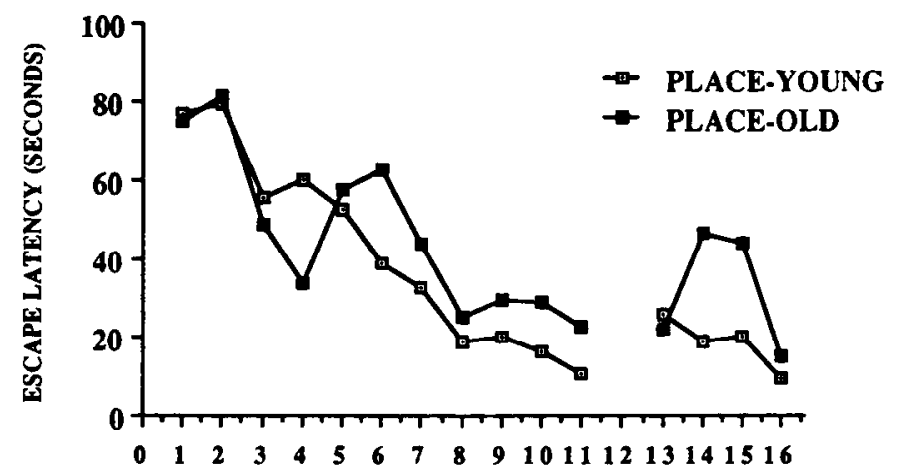

Figure 4. Escape latencies of old and young rats place-trained for $4 \mathrm{~d}$. 


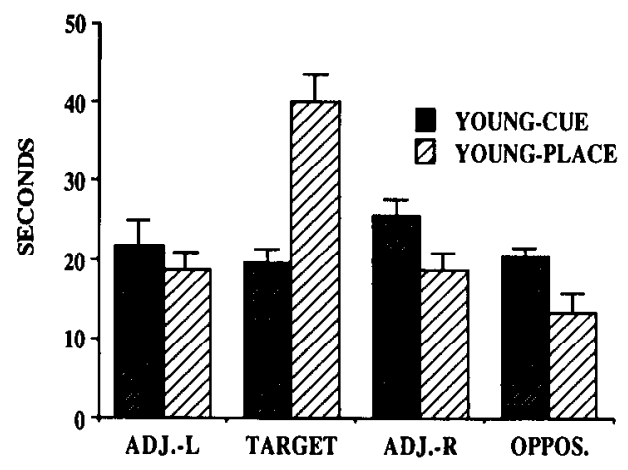

QUADRANT

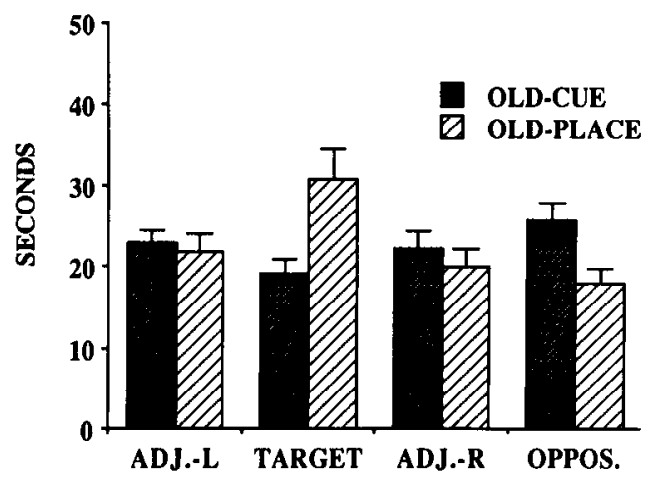

QUADRANT

Figure 5. Time spent in each of the quadrants during the probe trial for young and old rats either cue-trained or place-trained for $4 \mathrm{~d}$. Placetrained rats spent significantly more time in the target quadrant than did cue-trained rats, but this effect is more prominent among young rats.

quadrant. For place-trained rats, this quadrant is where the platform was located during training, and for cue-trained rats, which had not heen trained to a particular location, the target is the quadrant that bears the same relationship to the entry point as that used for place-trained animals. Young cue-trained animals tended to distribute their search evenly across all quadrants, but all 9 young place-trained animals spent more time in the target quadrant than in any other. This spatial bias shown by the place-trained animals is a common feature of this task and serves to demonstrate that place-trained animals do not use cues associated with the platform itself (e.g., they cannot see it), but instead have learned its location on the basis of spatial information available in the room.

The relative degree of spatial bias exhibited by young and old place-trained animals was evaluated by analyzing the time spent in the target quadrant using a 2-way ANOVA for age and training condition. This analysis reveals a significant overall spatial bias among place-trained rats, as these animals spent significantly more time in the target quadrant than did cue-trained animals $(F(1,30)=21.77, p<0.001)$, but this tendency is not as marked for old animals as it is for young animals, which results in a significant training condition $x$ age interaction $(F(1,30)=4.17, p<0.05)$. Thus, it is clear from the measure of quadrant time during the probe trial that place-training does not produce the same degree of spatial bias in old as in young animals. This is further supported by a consideration of the

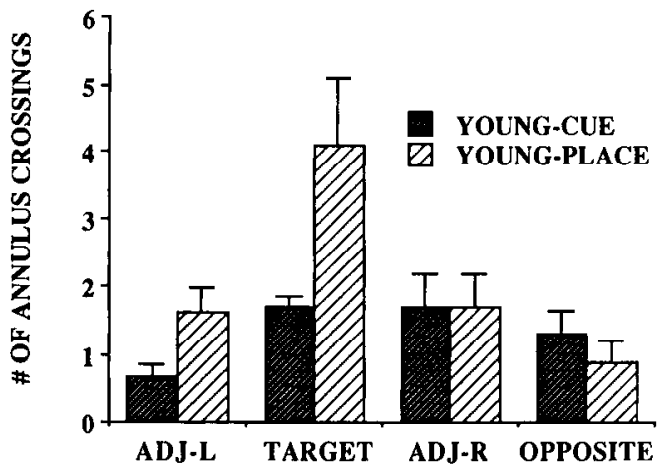

QUADRANT

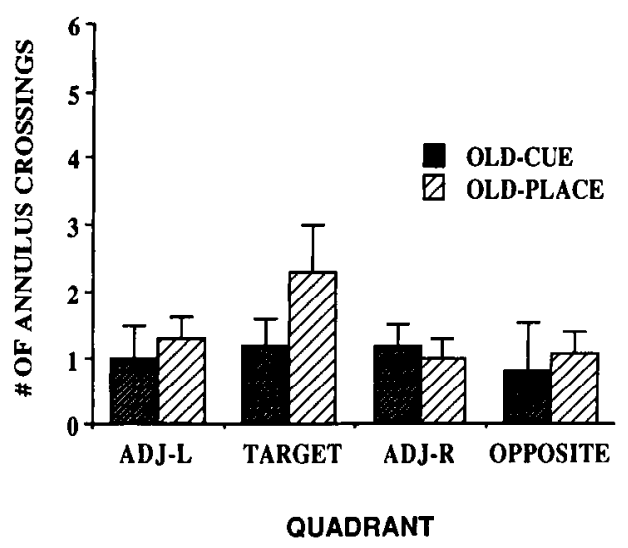

Figure 6. Number of annulus crossings in each of the quadrants during the probe trial for young and old rats either cue-trained or place-trained for $4 \mathrm{~d}$. Young place-trained rats crossed the target annulus significantly more often than did young cue-trained rats. This effect was not significant for old rats.

annulus crossing data found in Figure 6. Here, we find increased target annulus crossings after place-training (relative to those seen after cue-training) in young ( $p<0.025)$, but not in old, rats.

It thus appears that at the time of the probe trial (trial 12), place-trained aged rats have less secure knowledge of the normal location of the training platform and therefore spend less time searching in the vicinity of that location during the probe trial. To the degree that behavioral measures such as quadrant time and annulus crossings during the probe trial reflect the extent to which the task has been acquired, it can be said that placetrained old animals at this stage of training have not achieved the same level of mastery as their younger counterparts. Consistent with this age-related deficit in the acquisition of spatial information is a tendency that approaches significance for old place-trained animals to have somewhat longer escape latencies than young animals on the trials immediately preceding the free $\operatorname{swim}(F(1,16)=4.09, p<0.10$, for trials $9-11)$. In contrast, at this point in training, the escape latencies of old and young cuetrained animals are indistinguishable (Fig. 3).

As discussed earlier, a significant decrease in hippocampal HACU in place-trained young animals was observed after $4 \mathrm{~d}$ of training. HACU was also measured at this time point in aged animals to see if training-induced changes in HACU were blunted in parallel to the age-related deficits observed in spatial performance. From the results of this experiment, displayed in 
UPTAKE AT 0.4UM CHOLINE-HIPPOCAMPUS FOUR DAYS OF TRAINING

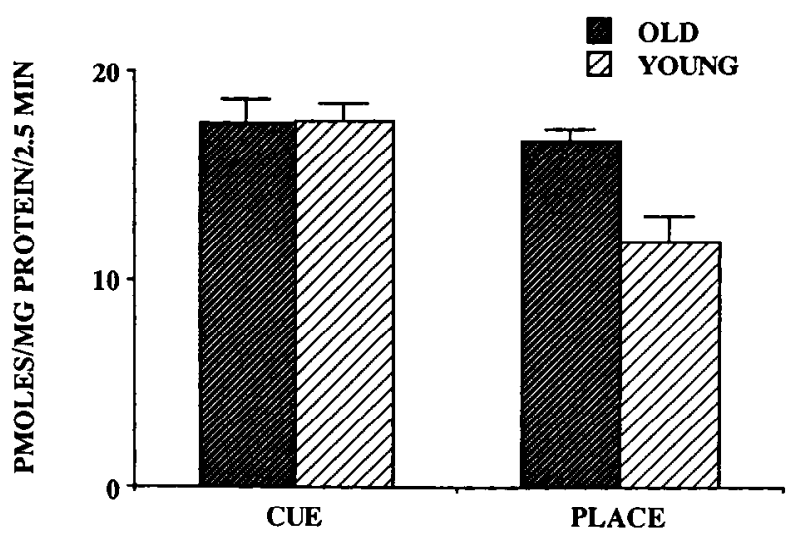

Figure 7. High-affinity choline uptake in hippocampus at $0.4 \mu \mathrm{M}$ choline for young and old rats either place-trained or cue-trained for $4 \mathrm{~d}$ (Experiments 2 and 3). The training-induced change in hippocampal uptake apparent in young animals is not observed in old animals.

Figure 7, it is clear that the training-induced decrease in hippocampal HACU seen in young animals is not found in aged animals. In addition, we observed no task-related change in HACU in the cortex of aged rats (data not shown). Thus, aged animals, which displayed impaired aquisition of this spatial task, did not show the training-induced change in hippocampal HACU that was seen in young animals, which were more proficient at this task.

$V_{\max } \mathrm{s}$ for hippocampal HACU in the old cue-trained and placetrained rats were $39.14 \pm 3.79$ and $37.48 \pm 2.89 \mathrm{pmol} / \mathrm{mg}$ protein $/ 2.5 \mathrm{~min}$, respectively; $K_{\mathrm{m}} \mathrm{s}$ were $0.49 \pm 0.04$ and $0.50 \pm$ $0.06 \mu \mathrm{M}$, respectively. Thus, hippocampal HACU in these old trained rats does not appear to be any different from that observed in the experimentally naive animals from Experiment 1 or in young rats that were killed after $4 \mathrm{~d}$ of cue-training.

Interestingly, one place-trained aged animal showed an apparent training-induced decrease in hippocampal HACU (i.e., a level of HACU at $0.4 \mu \mathrm{M}$ more than 1 SD below the mean for young, naive controls), and inspection of its free swim data shows the clearest example of a spatial bias among the aged animals (see Fig. 8). This suggests the possibility that the course of acquisition during place-training might be closely coupled to the training-induced change in hippocampal HACU. We explored this possibility in an additional experiment with young animals.

\section{Experiment 4}

In this experiment, the spatial environment surrounding the maze was degraded for some animals by removing some objects from the room and moving others farther from the maze. In this way we were able to produce relatively slower acquisition of place-training in some young animals, as measured by the quadrant time measure obtained during the probe trial on day 3 . This greater variability of performance among young animals allowed us to explore the relationship between acquisition rate and HACU on day 4. Also, some animals in this experiment received 4 additional days of training. This additional training served as an extension of the acquisition phase for slow learners and as overtraining for fast learners. HACU was measured at

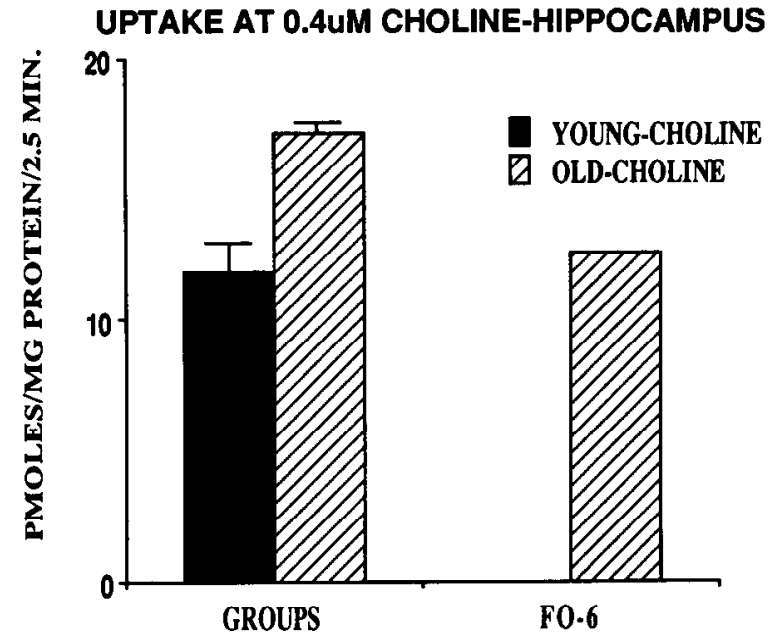

TARGET QUADRANT TIME
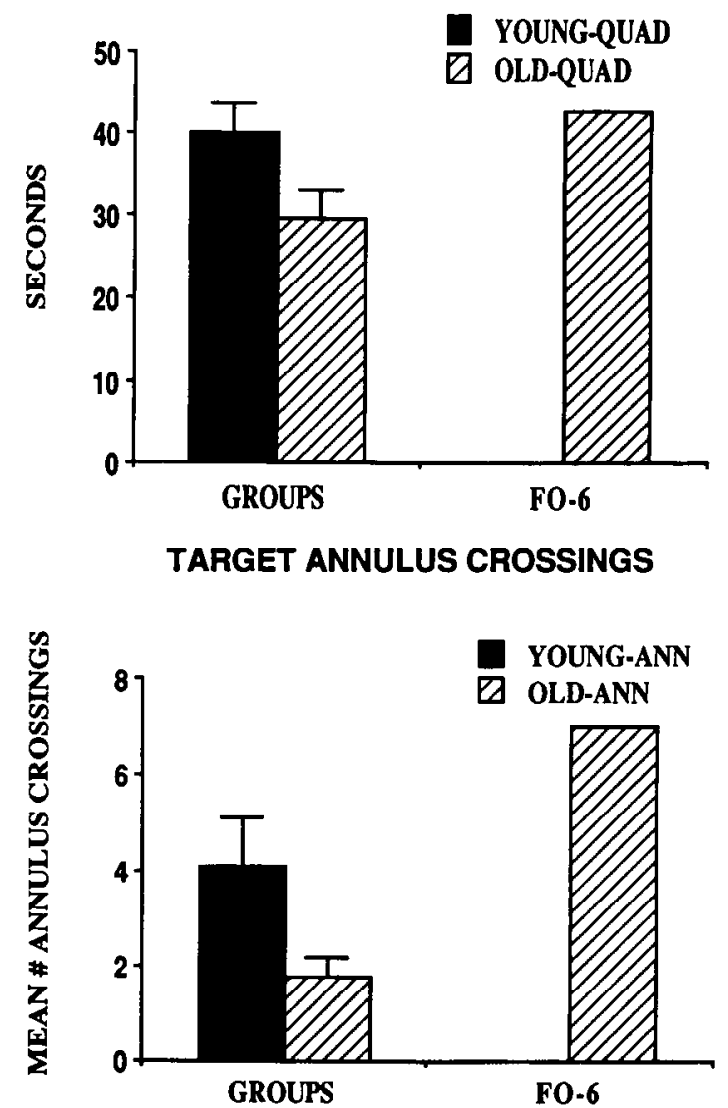

Figure 8. Comparison of the best learner among the old place-trained rats (FO-6) with the other old rats and the young rats place-trained for $4 \mathrm{~d}$ on measures of hippocampal high-affinity choline uptake at $0.4 \mu \mathrm{M}$ choline, time in training quadrant during the probe trial, and number of annulus crossings in target quadrant during the probe trial.

$0.4 \mu \mathrm{M}$ choline on day 4 or day 8 in fast and slow learners, and for each animal the HACU measured could be compared to that obtained from its own yoked control animal. Animals were again killed 15 min after the last training session, and HACU was determined in parietal cortex and hippocampus, as in the previous experiments, as well as in medial prefrontal cortex.

$\mathrm{HACU}$ in these brain regions for fast and slow learners was 


\section{FOUR DAYS OF TRAINING}

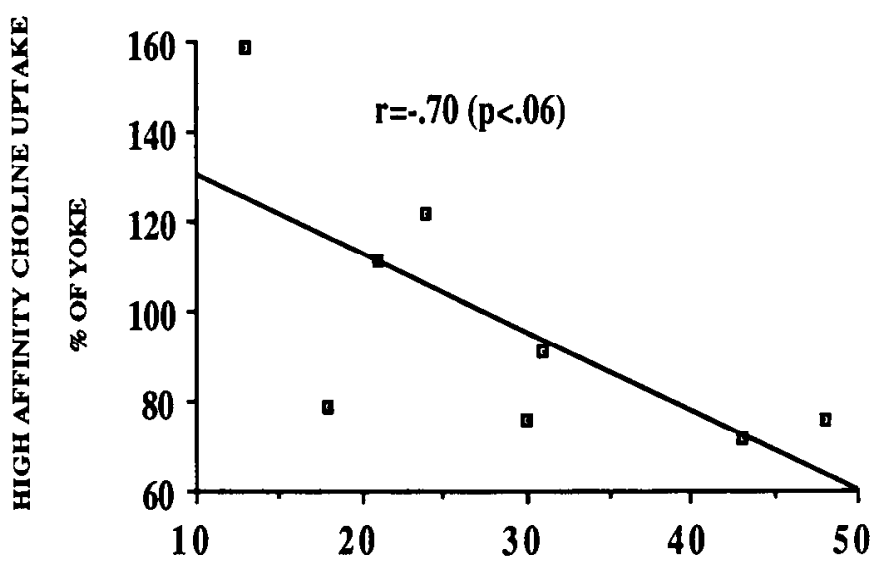

TRAINING QUADRANT TIME

\section{EIGHT DAYS OF TRAINING}

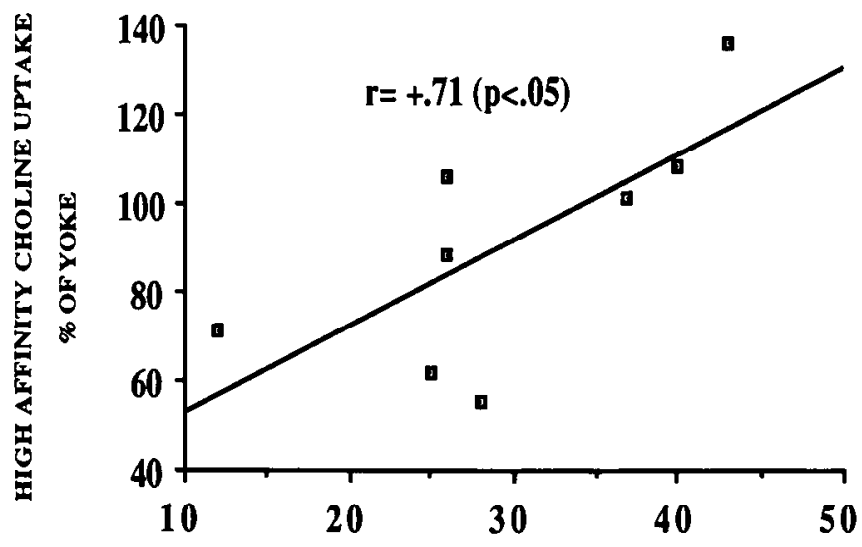

TRAINING QUADRANT TIME

Figure 9. Scatter plots of high-affinity choline uptake in hippocampus at $0.4 \mu \mathrm{M}$ choline (expressed as the percentage of yoked control uptake) versus the time in target quadrant during the probe trial for rats placetrained for either $4 \mathrm{~d}$ (above) or $8 \mathrm{~d}$ (below).

comparcd as a function of amount of training and training condition (place vs yoke). We used quadrant time data obtained during the day 3 probe trial to determine the learning rate of the place-trained animals. On the basis of the probe trial data from the young cue-trained animals tested in Experiment 2, we had determined that $30 \mathrm{sec}$ was the upper bound of the $90 \%$ confidence interval. Thus, since only $5 \%$ of animals that presumably have not developed a spatial bias would be expected to display a training quadrant time of $30 \mathrm{sec}$ or more, we established this as our cutoff between "fast" and "slow" learners a priori. Using these designations, a 3-way ANOVA for learning rate, training condition, and days of training was conducted for $\mathrm{HACU}$ in each brain region.

This analysis reveals no significant main effects on hippocampal HACU, but a significant 3-way interaction between learning rate, training condition, and amount of training $(F(1,24)=7.40$, $p<0.02$ ). This interaction appears to result from the tendency for slow learners to show decreased hippocampal HACU compared to yoked control animals after $8 \mathrm{~d}$ of place-training, and for fast learners to show this same change earlier in training. The significant decrease in hippocampal HACU (relative to yoked controls) among fast learners after $4 \mathrm{~d}$ of training $(t(6)=3.14$, $p<0.025$ ) is consistent with our demonstration in Experiment 2 that place-training reduces hippocampal HACU after $4 \mathrm{~d}$ in young animals proficient at the task. The discovery that the reverse relationship between acquisition rate and HACU exists after $8 \mathrm{~d}$ of training in the current experiment suggests that the altered hippocampal HACU induced by place-training is only characteristic of the acquisition phase of training. Thus, fast learners show a decrease in hippocampal HACU at day 4 , during the acquisition period, but not at day 8 , during the overtraining period. In contrast, slow learners show the decrease in hippocampal HACU at day 8 , which is presumably still within their acquisition period.

A scatter plot of this relationship can be used to illustrate the interaction. In Figure 9, we have plotted the relationship between quadrant time (our index of acquisition during placetraining, obtained for all animals on the day 3 probe trial) and hippocampal HACU (expressed as a percentage of yoked control) for animals trained for either 4 or $8 \mathrm{~d}$. Linear-regression analysis reveals a nearly significant negative relationship between hippocampal HACU and quadrant time after $4 \mathrm{~d}$ of training $(r=-0.70, p<0.06)$ but a significant positive relationship between these 2 measures after $8 \mathrm{~d}$ of training $(r=+0.71$, $p<0.05)$.

Analysis of HACU in parietal cortex in this experiment reveals a significant main effect of training condition $(F(1,24)=$ $5.02, p<0.05$ ). HACU in this region was significantly lower in place-trained animals than in their yoked controls regardless of the amount of training or the rate of learning, as no significant interaction terms involving this variable were found. By contrast, HACU in medial prefrontal cortex was not differentially affected by the training conditions in this experiment, but did increase significantly between 4 and $8 \mathrm{~d}$ of training, resulting in a significant main effect of length of training $(F(1,24)=8.57$, $p<0.01)$. For place-trained animals in this experiment, we noted a statistically significant relationship between the change in HACU relative to yoked control in parietal cortex and that observed in the same animals in hippocampus $(r=+0.69, p<$ 0.002 ). This is consistent with the relationship in these regions, demonstrated in Experiment 2, between HACU after cue- and place-training. In contrast, the change in HACU relative to yoked controls in medial prefrontal cortex and hippocampus was not significantly correlated in these animals $(r=+0.10)$.

\section{Discussion}

There are 2 major results from this study. This first is the demonstration and characterization of a task-specific, training-induced change in hippocampal HACU in young animals. The second notable finding is the failure to observe this change in behaviorally impaired old animals. Thus, we have established a direct, albeit still correlational, link between training on a task that is disrupted by cholinergic blockade and a functional change in the cholinergic system of young animals. Further, our discovery that this change does not occur in old animals that display impaired performance on this task is consistent with the cholinergic hypothesis of memory deficits in aging. 


\section{$H A C U$ in young rats}

Changes in HACU in hippocampus and cortex are regarded as an indication of altered cholinergic activity in these regions (for review, see Kuhar and Murin, 1978). This interpretation is based on a body of research demonstrating parallel shifts in ACh turnover and $\mathrm{HACU}$ in cortex and hippocampus following a varicty of pharmacological manipulations. We propose, therefore, that the reduction in hippocampal HACU that we observed following training reflects a decrease in the activity of the septohippocampal cholinergic system at the time of death. Since rats were killed $15 \mathrm{~min}$ following the training session, decreased HACU at this time point does not necessarily imply that cholinergic activity is reduced during the training session. Indeed, biphasic changes in HACU have been reported. A 10 min episode of immobilization restraint increases HACU into hippocampal synaptosomes (Finkelstein et al., 1985), but decreased HACU relative to control is noted when the episode is prolonged (45 min) or repeated (Finkelstein et al., 1985; Lai et al., 1986). The training-induced HACU decrease observed in our experiments when animals are killed after training could likewise reflect a compensatory reduction in cholinergic activity following a period of activation.

Although activation of the pituitary/adrenocortical axis (Gilad et al., 1985) affects HACU in a manner similar to that we observed after training, it is unlikely that a corticosterone response can account for our HACU data. First, immobilization stress produces parallel changes in HACU in hippocampus and frontal cortex (Lai et al., 1986), whereas in the present experiments we noted no difference in IIACU as a function of training condition in medial prefrontal cortex. Second, we designed our experiments to distinguish the effects of physical stress; hence the use of yoked controls. Third, animals in Experiment 2 that trained for only $1 \mathrm{~d}$ spent more time in the pool on the day they were killed than did animals trained for $4 \mathrm{~d}$, but differences in HACU were not noted among animals trained for $1 \mathrm{~d}$. Finally, the serum corticosterone determined in animals in Experiment 4 (M. A. Pelleymounter et al., unpublished observations) was not correlated with HACU in any of the 3 brain regions considered. It is reasonable, therefore, to interpret the difference in HACU as being related to the specific learning strategies required-spatial mapping versus cue location - and not to generalized physical stress effects.

Altered HACU in the hippocampus of young animals only occurred following place-training on the water maze, a task that has previously been shown to be disrupted both by hippocampal damage and by muscarinic blockade. In contrast, cue-training on the water maze, which is not affected by these manipulations, was not accompanied by any change in hippocampal HACU. This task specificity differs from the behaviorally induced change in hippocampal HACU observed by Wenk et al. (1984). They found that training on tasks with different sensitivities to hippocampal damage and muscarinic blockade had equivalent effects on hippocampal HACU. Thus, they did not observe a correspondence between the presumed involvement of the hippocampus and the cholinergic system in the performance of the tasks they used in the subsequently measured change in hippocampal HACU. In contrast, the altered hippocampal HACU we observed was specifically associated with training on a task that is apparently dependent on cholinergic and hippocampal function. This task-specific modification of HACU would ap- pear to provide strong support for a functional link between the observed change in HACU and the training.

Further evidence for a functional link between the observed change in HACU following place-training and the performance of this task is provided in Experiment 4. In this experiment, we demonstrated that the training-induced decrease in hippocampal HACU is related to the rate of acquisition. Further, the training-induced change appears to be limited to the acquisition period. This suggests that the effect on hippocampal HACU is not due so much to performance of the task as it is to its acquisition. Interestingly, there appears to be a temporal match between the period during which we observed training-induced changes in HACU and the period of maximum sensitivity to cholinergic blockade on this task. Whishaw (1985) and Buresova et al. (1986) have both found that doses of muscarinic antagonists that impair the acquisition of the place version of the task do not disrupt the performance of well-trained animals. Thus, the acquisition period is characterized both by sensitivity to muscarinic blockade and by training-induced changes in hippocampal HACU.

The failure to observe training-induced reduction in hippocampal HACU beyond the acquisition period might also explain the most obvious difference between our findings and those of Wenk et al. (1984). Rather than the training-induced decrease in HACU that we typically saw during acquisition, they noted increased hippocampal HACU in rats that had received 4 weeks of training on the radial-arm maze. Since we also observed a trend toward higher hippocampal HACU in overtrained animals (Fig. 9), both studies suggest that extensive training on spatial tasks results in increased hippocampal HACU. However, our work also suggests that training during the acquisition phase has quite a different effect on hippocampal HACU. Further, we should note that the radial-arm maze procedure primarily taps spatial working memory, whereas place-training in the water maze is a reference memory task. Thus, our results are not necessarily imcompatible with those of Wenk et al. (1984).

Our observation that a change in HACU subsequent to training on a spatial memory task was most reliably found in the hippocampus is consistent with a body of literature implicating the hippocampus as a structure important in the formation of spatial maps (O'Keefe and Nadel, 1978). The parietal cortex has also been proposed as a structure important in the formation of cognitive maps (Kesner, 1986). It is perhaps not surprising, then, that the level of HACU we observed in the hippocampus subsequent to behavioral training was closely related to that observed in the parietal cortex. Parietal cortex and hippocampus may, however, respond to different aspects of place-training, which could explain the somewhat distinctive effects of placetraining on HACU in these 2 regions. In Experiment 4, we noted a statistically significant correlation between the effects of placetraining on $\mathrm{HACU}$ in hippocampus and those observed in parietal (but not medial prefrontal) cortex. Unlike the traininginduced decrease in hippocampal $\mathrm{HACU}$, which was related to the rate of acquisition and the amount of training, however, training-induced decreases in $\mathrm{HACU}$ in parietal cortex were observed in nearly all place-trained animals (relative to their yoked controls) in this experiment. Retrieval of spatial information represented in parietal cortex during place-training could be accompanied by altered cholinergic activity. This would be similar to the cholinergically activated retrieval mechanism proposed by McGeer (1984) and would be consistent with the im- 
portance of basal forebrain cholinergic input to cortex for retrieval of spatial information (Murray and Fibiger, 1986). The septohippocampal cholinergic system, in contrast, might be engaged primarily when animals are initially learning to identify motivationally relevant features of a spatial environment (i.e., the location of the escape platform). This distinction is similar to that proposed by Kesner (1986), who suggests that the spatial map is stored within parietal cortex but is established via hippocampal circuitry, and with the demonstration that electrophysiological manipulation of hippocampal function interferes with learning a new spatial task, but not with the performance of a previously acquired spatial task (McNaughton et al., 1986).

\section{Behavioral data from aged rats}

Examination of the escape latencies of old and young animals reveals an age effect of borderline statistical significance. Older animals tended to take longer to reach the escape platform than did younger animals. This trend could, in part, be related to the diminished exploratory activity often characteristic of old animals. We observed that old animals tended to spend much more time scrabbling around the edges of the pool. Interestingly, this propensity for scrabbling is also characteristic of atropine-treated young animals (Whishaw, 1985). This behavior may account for the rather long latencies seen in old cue-trained animals during early trials (see Fig. 3). Consistent with this interpretation is the finding by Rapp et al. (1987) that old animals that are initially place-trained, and thus well-habituated, show no agerelated deficit when subsequently cue-trained.

We also found that place-trained aged animals spent significantly less time in the vicinity of the training platform location during the free swim than did their younger counterparts. Although older animals displayed some knowledge of the platform location during the free swim, their "spatial map" was significantly less precise. This deficit is dependent on age, as distinct from the history of the aged animals: a previous report from our laboratory determined that middle-aged rats (12 months at the time of testing) obtained as retired breeders are not impaired in either place or cue learning in the water maze (Rapp et al., 1987). On trials immediately before the free swim, the escape latencies of old rats tended to be somewhat longer than those of young animals, but despite an impairment in spatial mapping, old animals in the present experiment substantially improved their escape latencies during the course of place-training. As with many spatial memory tasks, spatial mapping is perhaps the most efficient, but not the only possible, strategy during place-training on the water maze. Since old rats tend to choose strategies that do not rely on spatial mapping when several are available (Barnes et al., 1980), our old rats may have been using alternative strategies, such as swimming around the pool at a fixed distance from the wall.

\section{HACU in aged rats}

We did not observe any age-related difference in either the $K_{\mathrm{m}}$ or $V_{\max }$ of HACU in the hippocampus of experimentally naive animals (Experiment 1). Sherman et al. (1981) did note a small, but significant, decrease in hippocampal HACU in Fischer 344 rats, but since we used Long-Evans rats, a strain difference is a possible explanation for this discrepancy. Our failure to find an age effect on HACU in hippocampus in Long-Evans animals is consistent with Wheeler's failure to note any effect of age on HACU in the cortex of this same strain (Wheeler, 1985). Despite frequent claims of diminished cholinergic function during aging, several studies have failed to reveal consistent age-related changes in other measures of cholinergic integrity, including ChAT activity, muscarinic binding, and basal in vitro $\mathrm{ACh}$ release, in hippocampus and cortex of experimentally naive rodents (Meek et al., 1977; Morin and Wasterlain, 1980; Ingram et al., 1981; Pedata et al., 1983; Meyer et al., 1984). This does not necessarily imply that the response of the aged cholinergic system to appropriate stimulation is normal; hence, our interest in the response of this system to behavioral challenge.

The specific relationship between place-training and hippocampal HACU found in young animals is not characteristic of old animals. Because we also find an age-related impairment in the development of a spatial bias during place-training, we have discovered an age-related behavioral deficit that coincides with an age-related disruption in cholinergic function. It is not likely that this failure to find a training-induced alteration in HACU in aged rats is due to a generalized deficit in the regulation of HACU in the aged brain, as Sherman et al. (1981) demonstrated that depolarization of hippocampal synaptosomes induces an equivalent increase in subsequently measured $\mathrm{HACU}$ in old and young rats. If a training-induced decrease in HACU represents a compensatory reduction in cholinergic activity following its initial activation, as we have previously suggested, the blunted response of HACU in old animals could suggest either a diminished activation of the septohippocampal cholinergic system during learning or that the compensatory mechanism responsible for the biphasic response of the cholinergic system is not fully functional in old animals.

An age-related failure to engage the cholinergic system during place-training could, then, account for the poor spatial mapping in old animals. It is also possible, however, that the delay in the development of spatial mapping in old animals is the cause, rather than the result, of altered cholinergic activation. Such a relationship between task competence and cholinergic activity is actually consistent with our finding that when environmental manipulations delay acquisition in young animals, the development of training-induced changes in HACU is similarly delayed. Thus, since old animals eventually attain the level of spatial bias seen in young animals (Rapp et al., 1987), hippocampal HACU changes might occur at some later point in the course of their training. Alternatively, behaviorally impaired old rats may not show the training-induced change in HACU even after they learn the task. Such a result could be obtained if the septohippocampal cholinergic system modulates, but is not necessary for, the acquisition of spatial information or if old animals eventually learn the task by using some less efficient strategy that does not engage the septohippocampal cholinergic system. Consistent with this latter possibility is Whishaw's (1985) suggestion that atropine-treated young rats that eventually learn this task do so via just such an alternative strategy.

While we have identified a change in HACU that appears to be linked to acquisition of a learning and memory task in young animals, the interpretation of what this represents in terms of training-induced changes in cholinergic activity will require further study. It is clear, however, that this change in cholinergic function does not accompany training in old animals that show impaired acquisition. Thus, we have demonstrated a link between altered responsivity of the cholinergic system and behavioral deficits in aged animals. This finding is consistent with the cholinergic hypothesis, but the elucidation of the causal relationship between these age-related phenomena, if indeed there is one, will require additional investigation. 


\section{References}

Atweh, S., J. R. Simon, and M. J. Kuhar (1975) Utilization of sodium dependent high affinity uptake in vitro as a measure of activity of cholinergic neurons in vivo. Life Sci. 17: 1535-1544.

Barnes, C. A., L. Nadel, and W. K. Honig (1980) Spatial memory deficits in senescent rats. Can. J. Psychol. 34: 29-39.

Bartus, R. T., R. L. Dean, B. Beer, and A. S. Lippa (1982) The cholinergic hypothesis of geriatric memory dysfunction. Science 217 : 408-417.

Bartus, R. T., R. L. Dean, M. J. Pontecorvo, and C. Flicker (1985) The cholinergic hypothesis: A historical overview, current perspective, and future directions. Ann. NY Acad. Sci. 444: 332-358.

Burcsova, O., J. J. Bolhuis, and J. Bures (1986) Differential effects of cholinergic blockade on performance of rats in the water tank navigation task and in a radial water maze. Behav. Neurosci. 100: 476482.

Consolo, S., J.-X. Wang, F. Fiorentini, A. Vezzani, and H. Ladinsky (1986) In vivo and in vitro studies on the regulation of cholinergic neurotransmission in striatum, hippocampus and cortex of aged rats. Brain Res. 374: 212-218.

Cooper, D. O., D. E. Schmidt, and R. J. Barrett (1983) Strain specific cholinergic changes in response to stress: Analysis of a time-dependent avoidance variation. Pharmacol. Biochem. Behav. 19: 457-462.

Davis, H. P., A. Idowu, and G. E. Gibson (1983) Improvement of 8-arm maze performance in aged Fischer 344 rats with 3,4-diaminopyridine. Exp. Aging Res. 9: 211-213.

Decker, M., M. Pcllcymounter, R. Cody, and M. Gallagher (1986) Altered high affinity choline uptake with "place" but not "cue" version of the Morris water maze. Soc. Neurosci. Abstr. 12: 716.

Dokla, C. P., L. J. Thal, E. L. Gardner, D. Hann, J. C. Passabet, L. Fitzgerald, and L. Leiman (1985) Ibotenic-acid lesions of the nucleus basalis of Maynert impair spatial-reference in the Morris water task. Soc. Neurosci. Abstr. 11: 332.

Finkelstein, Y., B. Koffler, J. M. Rabey, and G. M. Gilad (1985) Dynamics of cholinergic synaptic mechanisms in rat hippocampus after stress. Brain Res. 343: 314-319.

Gage, F. H., and A. Björklund (1986) Cholinergic septal grafts into the hippocampal formation improve spatial learning and memory in aged rats by an atropine-sensitive mechanism. J. Neurosci. 6: 28372847.

Gage, F. H., A. Björklund, U. Stencvi, S. B. Dunnett, and P. A. T. Kelly (1984a) Intrahippocampal septal grafts ameliorate learning impairments in aged rats. Science 225: 533-536.

Gage, F. H., S. B. Dunnett, and A. Björklund (1984b) Spatial learning and motor deficits in aged rats. Neurobiol. Aging 5: 43-48.

Gilad, G. M., B. D. Mahon, Y. Finkelstein, B. Koffler, and V. H. Gilad (1985) Stress-induced activation of the hippocampal cholinergic system and the pituitary-adrenocortical axis. Brain Res. 347: 404-408.

Gold, P. E., and S. F. Zornetzer (1983) The mnemon and its juices: Neuromodulation of memory processes. Behav. Neural Biol. 38: 151189.

Ingram, D. K., E. D. London, and C. L. Goodrick (1981) Age and neurochemical correlates of radial maze performance in rats. Neurobiol. Aging, 2: 41-47.

Kesner, R. P. (1986) Neurobiological vicws of mcmory. In Learning and Memory: A Biological View, J. L. Martinez, Jr. and R. P. Kesner, eds., pp. 399-438, Academic, Orlando, FL.

Kuhar, M. J., and L. C. Murin (1978) Sodium-dependent, high affinity choline uptake. J. Neurochem. 30: 15-21.

Lai, H., J. Zabawska, and A. Horita (1986) Sodium dependent, highaftinity choline uptake in hippocampus and frontal cortex of the rat affected by acute restraint stress. Brain Res. 372: 366-369.

Lowry, O. H., N. J. Rosebraugh, A. L. Farr, and R. J. Randal (1951) Protein measurement with the Folin reagent. J. Biol. Chem. 193:265275.

Luine, V. N., K. J. Renner, S. Heady, and K. J. Jones (1986) Age and sex-dependent decreases in ChAT in basal forebrain nuclei. Neurobiol. Aging 7: 193-198.

McGeer, P. L. (1984) The 12th J. A. F. Stevenson memorial lecture.
Aging, Alzheimer's disease and the cholinergic system. Can. J. Pharmacol. 62: 741-754.

McNaughton, B. L., C. A. Barnes, G. Rao, J. Baldwin, and M. Rassmussen (1986) Long-term enhancement of hippocampal synaptic transmission and the acquisition of spatial information. J. Neurosci. 6: 563-571.

Meek, J. L., L. Bertilsson, D. L. Cheney, G. Zsilla, and E. Costa (1977) Aging-induced changes in acetylcholine and serotonin content of discrete brain nuclei. J. Gerontol. 32: 129-131.

Meyer, E. M., E. St. Onge, and F. T. Crews (1984) Effects of aging on rat cortical presynaptic cholinergic processes. Neurobiol. Aging 5 . 315-317.

Morin, A. M., and C. G. Wasterlain (1980) Aging and rat brain muscarinic receptors as measured by quinuclidinyl benzilate binding. Neurochem. Res. 5: 301-308.

Morris, R. G. M. (1981) Spatial localization does not require the presence of local cues. Learn. Motiv. 12: 239-260.

Morris, R. G. M., P. Garrard, J. N. P. Rawlins, and J. O'Keefe (1982) Place navigation impaired in rats with hippocampal lesions. Nature 297: 681-683.

Murray, C. L., and H. C. Fibiger (1986) Pilocarpine and physostigmine attenuate spatial memory impairments produced by lesions of the nucleus basalis magnocellularis. Behav. Neurosci. 100: 23-32.

O'Keefe, J., and L. Nadel (1978) The Hippocampus as a Cognitive Map, Clarendon Press, Oxford, UK.

Pedata, F., J. Slavikova, A. Kotas, and G. Pepeu (1983) Acetylcholine release from rat cortical slices during postnatal development and aging. Neurobiol. Aging 4: 31-35.

Perry, E. K. (1980) The cholinergic system in old age and Alzheimer's disease. Age and Ageing 9: 1-8.

Rapp, P. R., R. A. Rosenberg, and M. Gallagher, M. (1987) An evaluation of spatial information processing in aged rats. Behav. Neurosci. 101: 3-12.

Sherman, K. A., M. J. Zigmond, and I. Hanin (1978) High affinity choline uptake in striatum and hippocampus: Differential effects of treatments which release acetylcholine. Life Sci. 23: 1863-1870.

Sherman, K. A., J. E. Kuster, R. L. Dean, R. T. Bartus, and E. Friedman (1981) Presynaptic cholinergic mechanisms in brain of aged rats with memory impairments. Neurobiol. Aging 2: 99-104.

Simon, J. R., and M. J. Kuhar (1975) Impulse-flow regulation of high affinity choline uptake in brain cholinergic nerve terminals. Nature 255: $162-163$.

Simon, J. R., S. Atweh, and M. J. Kuhar (1976) Sodium-dependent high affinity choline uptake: A regulatory step in the synthesis of acetylcholine. J. Neurochem. 26: 909-922.

Squire, L. R., and H. P. Davis (1981) The pharmacology of memory: A neurobiological perspective. Annu. Rev. Pharmacol. Toxicol. 21: 323-356.

Sutherland, R. J., I. Q. Whishaw, and J. C. Regeher (1982) Cholinergic receptor blockade impairs spatial localization by use of distal cues in the rat. J. Comp. Physiol. Psychol. 96: 563-573.

Sutherland, R. J., I. Q. Whishaw, and B. Kolb (1983) A behavioral analysis of spatial localization following electrolytic, kainate- or colchicine-induced damage to the hippocampal formation in the rat. Behav. Brain Res. 7: 133-153.

Wenk, G., D. Hepler, and D. Olton (1984) Behavior alters the uptake of ${ }^{3} \mathrm{H}$-choline into acetylcholinergic neurons of the nucleus basalis magnocellularis and medal septal area. Behav. Brain Res. 13: 129138 .

Wheeler, D. D. (1985) Aging of membrane transport mechanisms in central nervous system - High affinity choline transport in rat cortical synaptosomes. Exp. Gerontol. 20:73-80.

Whishaw, I. Q. (1985) Cholinergic receptor blockade in the rat impairs locale but not taxon strategies for place navigation in a swimming pool. Behav. Neurosci. 99: 979-1005.

Whishaw, I. Q., W. T. O'Connor, and S. B. Dunnett (1985) Disruption of central cholinergic systems in the rat by basal forebrain lesion or atropine: Effects on feeding, sensorimotor behaviour, locomotor activity and spatial navigation. Behav. Brain Res. 17: 103-115. 